\title{
DIETAS Y CRECIMIENTO DE CRIAS DE TARICAYA Podocnemis uniflis (chalonia: Pelomedusidae) EN CAUTIVERIO, IQUITOS- PERU
}

Arturo Acosta Diaz *

Augusto Fachin Terán ${ }^{* *}$

\section{RESUMEN}

El trabajo se realizó de noviembre de 1994 a abril de 1995 en un estanque seminatural de Quistococha. Se experimentaron dietas usando Curimatus rutiloides $\mathrm{y}$ Curimatus sp. (peces); Musa paradisiaca, Manihot esculenta y Carica papaya. La dieta 1 fue omnívora (T1), la dieta 2 exclusivamente de carne de pescado (T2), la dieta 3 vegetariana (T3) y la dieta 4 fue también omnívora (T4). Las crías iniciaron el experimento con un peso promedio de $\mathrm{Ti} 16,45 \mathrm{~g}(\mathrm{~N} 12)$; T2=15,12 g (N12); $\mathrm{T} 3=15,00 \mathrm{~g}(\mathrm{~N} 12)$ y $\mathrm{T} 4=16,55 \mathrm{~g}(\mathrm{~N} 10)$.

Las crías del TI (N10), T2 (N8) y T4 $(\mathrm{N}=10)$ obtuvieron a los seis meses un peso promedio de 86,65 g, 93,06 g y 85,80 g, respectivamente, y las crías del T3 sólo pesaron $30,29 \mathrm{~g}$. El análisis de varianza fue significativo $(\mathrm{p}=0,05)$ para un $\mathrm{Ft}=8,55$ $\mathrm{y}$ un $\mathrm{Fc}=338,3$. Concluimos que una alimentación, comparada con una dieta vegetariana, que tiene como principal fuente de proteína al pescado, influye positivamente en el crecimiento de crías de taricaya Podocnemis unifilis en cautiverio.

Palabras clave: Dietas de crías, crecimiento.

* Profesor auxiliar- Departamento de Zoología. Universidad Nacional de la Amazonía Peruana (UNAP). Castilla postal 259. Teléfono 236121. Iquitos-Perú.

** $\quad$ Profesor auxiliar- Departamento de Zoología. Universidad Nacional de la Amazonía Peruana (UNAP). Castilla postal 259. Teléfono 236121. Iquitos-Perú. 


\section{ABSTRACT}

The study was realized from November 1994to April 1995, in seminatural pound at the Quistococha. The experimental diets consisted of Curimatus rutiloides and Curimatus sp. (fish), Alusa paradisíaca, Manihot sculenta, and Carica papaya. Diet 1 was omnivorous (TI), diet 2 was fihs only (T2), diet 3 was vegetarian (T3), and diet 4 was omnivorous (T4). The hatchlings started the experiment with following weights mean: $\mathrm{T} 1=16,45 \mathrm{~g}(\mathrm{~N}=12) ; \mathrm{T} 2=15,12 \mathrm{~g}(\mathrm{~N}=12) ; \mathrm{T} 3=15,00(\mathrm{~N}=12)$; and $\mathrm{T} 4=16,55 \mathrm{~g}(\mathrm{~N}=10)$.

The hatchlings of $\mathrm{T} 1(\mathrm{~N}=10), \mathrm{T} 2(\mathrm{~N}=8)$ and $\mathrm{T} 4(\mathrm{~N}=10)$ reached in 6 months mean weights of $86,65 \mathrm{~g}, 93,06 \mathrm{~g}$ and $85,80 \mathrm{~g}$, respectively, while those of T3 only reached $30,29 \mathrm{~g}$. Variance analysis was significant $(\mathrm{p}=0,05)$ for $\mathrm{Ft}=8,55$ and $\mathrm{Fc}=$ 338,3 . We conclude that, compared with a vegetarian diet, food containing fish as main protein source, has a positive effect on growth of Podocnemis unifilis hatchlings in captivity.

Key words: Hatchling diets, growth.

\section{INTRODUCCION}

La notoria disminución de las poblaciones de Podocnemis unifilis en la Amazonía Peruana hace necesario que su crianza en cautiverio sea estudiada para incentivar su cultivo con criterio técnico y conservacionista por parte del poblador amazónico y, de esa manera, evitar la extinción de esta valiosa especie.

Según Fachin et al. (1992), en los alrededores de Iquitos muchas personas crían taricayas, Podocnemis unifilis, en diferentes estadios de desarrollo, utilizando diversos tipos de insumos en su alimentación. Acosta et al. (1995) ensayaron dietas en crías de taricaya $(\mathrm{N}=9)$ de ocho meses de edad nacidas en cautiverio, determinándose que una alimentación omnívora en esta fase de desarrollo favorece su crecimiento.

Dentro de esta problemática el presente experimento tuvo como objetivo ensayar dietas alimenticias para verificar su influencia en el crecimiento de crías recién nacidas de Podocnemis unifilis en cautiverio en 1994. 


\section{MATERIALES Y METODOS}

El trabajo fue realizado en el Parque Turístico de Quistococha, ubicado en el kim 13,5 de la carretera Iquitos-Nauta, desde noviembre de 1994 hasta abril de 1995. La muestra experimental consistió en 46 crías de Podacnemis unifilis recién nacidas que fueron producidas por el proyecto de investigación «Cultivo y conservación de quelonios del género Podocnemis en estanques serninaturales» de la Universidad Nacional de la Amazonía Peruana.

Se usó el diseño con grupo de control pre y post test y el diseño estadístico usado fue el diseño completo al azar (DCA).

Las crías fueron distribuidas en cuatro sub-grupos: los tratamientos 1, 2, 3 iniciaron con 12 individuos cada uno de los cuales fue colocado en «jaulas» de $3 \mathrm{mx}$ 1,5 m ubicadas en la margen izquierda del estanque y construidas con mallas sintéticas; el tratamiento 4 inició con 10 individuos que fueron colocados en un estanque con un espejo de agua de $1026 \mathrm{~m}^{2}$. Los tratamientos se aplicaron durante seis meses y tuvieron las siguientes características:

Tratamiento 1 (Tl):denominado dieta omnívora con individuos solos y compuesto de «ractacara» Curimatus rutiloides y/o «llarnbino» Curimatus sp., «plátano maduro» Masa paradisiaca.

Tratamiento 2 (T2): denominado dieta carnívora con individuos solos y compuesto de Curimatus rutiloides y/o Curimatus sp.

Tratamiento 3 (T3): denominado dieta vegetariana con individuos solos y compuesto de «papaya» Carica papaya, «plátano» Masa paradisiaca y «yuca» Manihot esculenta.

Tratamiento 4 (T4): denominado dieta omnívora, junto a adultos, y compuesto de Curimatus rutiloides y/o Curimatus sp., Musa paradisíaca y otros frutos.

Los alimentos fueron suministrados diariamente de lunes a sábado, una vez al día. Los tratamientos 1, 2, y 3 recibieron $150 \mathrm{~g}$ de alimento diariamente. Antes de iniciar el experimento procedimos a tornar las siguientes medidas: largo y ancho del espaldar, largo del plastrón, ancho de la cabeza, altura del cuerno, largo y ancho de la placa femoral izquierda. Las medidas fueron tomadas con un calibrador Vernier (con precisión de $0,01 \mathrm{~mm}$ ) en línea recta y en el punto de mayor amplitud. Los especimenes fueron pesados durante los primeros meses con 
una pesola de $100 \mathrm{~g}$ con precisión de $1 \mathrm{~g}$ y cuando sus pesos fueron mayores se utilizó una pesola de $300 \mathrm{~g}$ con precisión de $2 \mathrm{~g}$. Los controles fueron individuales y una vez por mes y todos los individuos fueron marcados con un número correlativo en el caparazón con pintura esmalte color blanco. Los datos fueron procesados mediante el análisis de varianza (Anva) y la prueba de Duncan.

Se probó la siguiente hipótesis de trabajo:

El tipo de dieta (omnívora o carnívora) suministrado a las crías de $P$. unifilis y su aislamiento de los adultos, influye directamente en su crecimiento en condiciones de cautiverio.

\section{RESULTADOS Y DISCUSION}

\subsection{Crecimiento de crías de Podocnemis unifilis en cautiverio}

\subsubsection{Ganancia de peso y longitud}

El control pre-test lo realizamos en noviembre de 1994 y el último control en abril de 1995. Para analizar la influencia de las dietas en el crecimiento, sólo utilizamos el peso y la longitud del caparazón (medida en línea recta). El Cuadro 1 muestra el peso y longitud del caparazón de las crías antes de ser sometidas a las dietas.

Durante el desarrollo del experimento en el T2 murieron dos crías por causas desconocidas y otras dos se escaparon de la jaula; del T1 se escaparon dos crías y del T3 se escapó una cría. El Cuadro 2 muestra los pesos y medidas obtenidos al final del experimento.

Las crías del Ti, T2 y T4 obtuvieron a los cinco meses un peso total de 86,655 g, 93,06 g y 85,80 g y una longitud del caparazón de 8,17 cm, 8,28 cm y $8,06 \mathrm{~cm}$, respectivamente; mientras que las crías del T3 sólo lograron $30,29 \mathrm{~g}$ de peso y $5,37 \mathrm{~cm}$ de longitud del caparazón.

Las ganancias neta de peso y longitud del caparazón fueron: T1='70,20 g y $3,69 \mathrm{~cm} ; \mathrm{T} 2=77,94 \mathrm{~g}$ y $4,04 \mathrm{~cm}$; $\mathrm{T} 3=15,29 \mathrm{~g}$ y $1,18 \mathrm{~cm}$ y para $\mathrm{T} 4=$ 69,25 g y $3,62 \mathrm{~cm}$. 
Cuadro 1. Peso (P) y longitud del caparazón (LC) pre-test de crías de Podocnemis unifilis en condiciones de cautiverio. Quistococha. Noviembre 1994.

\begin{tabular}{|c|c|c|c|c|c|c|c|c|}
\hline \multirow{2}{*}{ Repeticiones } & \multicolumn{9}{|c|}{ Tratamientos } \\
\cline { 2 - 10 } & \multicolumn{2}{|c|}{ T1 } & \multicolumn{2}{c|}{ T2 } & \multicolumn{2}{c|}{ T3 } & \multicolumn{2}{c|}{ T4 } \\
\hline & $\begin{array}{c}\mathrm{P} \\
(\mathrm{g})\end{array}$ & $\begin{array}{c}\text { L.C } \\
(\mathrm{cm})\end{array}$ & $\begin{array}{c}\text { P } \\
(\mathrm{g})\end{array}$ & $\begin{array}{c}\text { L.C } \\
(\mathrm{cm})\end{array}$ & $\begin{array}{c}\text { P } \\
(\mathrm{g})\end{array}$ & $\begin{array}{c}\text { L.C } \\
(\mathrm{cm})\end{array}$ & $\begin{array}{c}\text { P } \\
(\mathrm{g})\end{array}$ & $\begin{array}{c}\text { L.C } \\
(\mathrm{cm})\end{array}$ \\
\hline 1 & 16,10 & 4,50 & 14,30 & 4,33 & 15,00 & 4,21 & 17,00 & 4,43 \\
\hline 2 & 16,20 & 4,48 & 15,50 & 4,10 & 13,00 & 3,95 & 16,50 & 4,45 \\
\hline 3 & 16,00 & 4,42 & 11,00 & 3,80 & 16,00 & 4,20 & 16,30 & 4,47 \\
\hline 4 & 17,20 & 4,55 & 14,00 & 3,93 & 15,00 & 4,27 & 16,30 & 4,43 \\
\hline 5 & 16,10 & 4,45 & 17,50 & 4,60 & 14,50 & 4,23 & 17,20 & 4,59 \\
\hline 6 & 17,00 & 4,57 & 17,00 & 4,70 & 14,20 & 4,12 & 17,00 & 4,48 \\
\hline 7 & 15,10 & 4,31 & 14,50 & 4,00 & 15,00 & 4,42 & 17,00 & 4,45 \\
\hline 8 & 17,50 & 4,55 & 14,20 & 4,05 & 14,20 & 4,18 & 17,00 & 4,47 \\
\hline 9 & 17,00 & 4,60 & 17,00 & 4,53 & 16,00 & 4,35 & 16,20 & 4,42 \\
\hline 10 & 17,00 & 4,61 & 16,50 & 4,48 & 15,50 & 4,15 & 15,00 & 4,25 \\
\hline 11 & 16,10 & 4,33 & 16,00 & 4,50 & 16,50 & 4,27 & --- & --- \\
\hline 12 & 16,20 & 4,42 & 14,00 & 4,95 & 15,20 & 4,20 & --- & --- \\
\hline Promedio & 16,45 & 4,48 & 15,12 & 4,24 & 15,00 & 4,19 & 16,55 & 4,44 \\
D.es. es* & 0,68 & 0,09 & 1,83 & 0,30 & 0,95 & 0,09 & 0,60 & 0,08 \\
\hline
\end{tabular}

* Desviación estándar.

Fuente: Proyecto Quelonios-UNAP.1994 


\section{Cuadro 2. Peso (P) y longitud del caparazón (LC) post-test de crías de} Podocnemis unfilis en condiciones de cautiverio. Quistococha. Abril 1995.

\begin{tabular}{|c|c|c|c|c|c|c|c|c|}
\hline \multirow{2}{*}{ Repeticiones } & \multicolumn{9}{|c|}{ Tratamientos } \\
\cline { 2 - 10 } & \multicolumn{2}{|c|}{ T1 } & \multicolumn{2}{c|}{ T2 } & \multicolumn{2}{c|}{ T3 } & \multicolumn{2}{c|}{ T4 } \\
\hline & $\begin{array}{c}\text { P } \\
(\mathrm{g})\end{array}$ & $\begin{array}{c}\text { L.C } \\
(\mathrm{cm})\end{array}$ & $\begin{array}{c}\text { P } \\
(\mathrm{g})\end{array}$ & $\begin{array}{c}\text { L.C } \\
(\mathrm{cm})\end{array}$ & $\begin{array}{c}\text { P } \\
(\mathrm{g})\end{array}$ & $\begin{array}{c}\text { L.C } \\
(\mathrm{cm})\end{array}$ & $\begin{array}{c}\text { P } \\
(\mathrm{g})\end{array}$ & $\begin{array}{c}\text { L.C } \\
(\mathrm{cm})\end{array}$ \\
\hline 1 & 79,00 & 7,92 & 87,00 & 8,40 & 40,00 & 6,20 & 94,00 & 8,25 \\
\hline 2 & 72,00 & 7,61 & 82,00 & 7,80 & 27,00 & 5,21 & 79,00 & 7,80 \\
\hline 3 & 93,00 & 8,18 & 111,00 & 9,10 & 26,50 & 5,10 & 96,00 & 8,55 \\
\hline 4 & 99,50 & 8,67 & 86,50 & 8,03 & 28,00 & 5,21 & 72,00 & 7,66 \\
\hline 5 & 84,00 & 8,06 & 69,00 & 7,20 & 20,50 & 4,74 & 67,00 & 7,32 \\
\hline 6 & 82,00 & 8,22 & 108,00 & 8,90 & 34,00 & 5,64 & 79,50 & 7,80 \\
\hline 7 & 90,00 & 8,30 & 105,00 & 8,73 & 37,50 & 5,76 & 96,00 & 8,57 \\
\hline 8 & 99,00 & 8,50 & 96,00 & 8,08 & 38,00 & 5,82 & 74,50 & 8,66 \\
\hline 9 & 85,00 & 8,12 & --- & --- & 28,20 & 5,28 & 97,00 & 8,34 \\
\hline 10 & 83,00 & 8,17 & --- & --- & 29,00 & 5,35 & 103,00 & 7,70 \\
\hline 11 & --- & --- & --- & --- & 24,50 & 4,80 & --- & --- \\
\hline & & & \multicolumn{7}{|c|}{} & & & & & \\
\hline Promedio & 8,17 & 93,06 & 8,28 & 30,29 & 5,38 & 85,80 & 8,06 & \\
D.est.* & 8,73 & 0,29 & 17,52 & 0,62 & 6,20 & 0,44 & 12,71 & 0,46 \\
\hline
\end{tabular}

* Desviación estándar.

Fuente:Proyecto Quelonios-UNAP. 1994.

La ganancia de peso y longitud del caparazón promedio mensual para los diferentes tratamientos fueron: $\mathrm{T} 1=14,04 \mathrm{~g}(\mathrm{D} . \mathrm{S}=5,1)$ y $0,73 \mathrm{~cm}(\mathrm{D} . \mathrm{S}=0,28)$; $\mathrm{T} 2=$ $15,58 \mathrm{~g}(\mathrm{D} . \mathrm{S}=7,9)$ y $0,80 \mathrm{~cm}(\mathrm{D} . \mathrm{S}=0,28)$; T3=3,05 g (D.S 0,59) y $0,23 \mathrm{~cm}$ (D.S $=$ $0,045)$ y para el T4=13,85 g (D.S 4,8$)$ y $0,72 \mathrm{~cm}(\mathrm{D} . \mathrm{S}=0,17)$.

El análisis de varianza fue significativo $(\mathrm{p}=0,05)$ para un $\mathrm{Ft} 8,55$ y un $\mathrm{Fc}=338,3$. Según la prueba de Duncan, los individuos sometidos a los Ti, T2 y T4 tuvieron una ganancia de peso y longitud del caparazón significativo $(\mathrm{p}=0,05)$ sobre los individuos del T3. Cabe resaltar que los individuos del T2 tuvieron una ganancia de peso $(93,06 \mathrm{~g})$ y longitud del caparazón $(8,28 \mathrm{~cm})$ significativo con relación a los individuos de los T1 $(86,65 \mathrm{~g} \mathrm{y} 8,17 \mathrm{~cm})$ y T4 $(85,80 \mathrm{~g}$ y 8,06$)$.

El aislamiento de las crías de taricaya en jaulas, evita la competencia por el alimento con individuos juveniles y adultos de la misma especie que se encuentran 
en el estanque; asimismo permite un mayor consumo del alimento por parte de las crías.

Por cuestiones económicas, de espacio y facilidad para lograr una captura rápida de los especimenes, se recomienda la aplicación del tratamiento 1. Asimismo, se observa que los especimenes de la dieta que contenía sólo carne de pescado obtuvieron mayor peso que la omnívora, pero el problema estaría en los costos y la dificultad para obtener el pescado en época de creciente en nuestra región, donde se observa una escasez de este recurso y mayor costo. Aunque la «ractacara» Curimatus rutiloides y el «llambino» Curimatus sp. son especies que presentan precios bajos en el mercado tanto en época de vaciante como en creciente lo que disminuiría los costos de alimentación. Los demás insumos utilizados se pueden obtener fácilmente en los puertos de la ciudad y principalmente en el área rural donde cada morador tiene su «platanal» (Musa paradisiaca), del cual se podría abastecer en caso de iniciarse una crianza intensiva de taricayas.

\section{AGRADECIMIENTO}

Agradecemos al Proyecto Cultivo y conservación de quelonios de agua dulce del género Podocnemis en estanques seminaturales de la Facultad de Ciencias Biológicas de la UNAP, por el apoyo financiero y logístico para la ejecución del presente trabajo.

\section{BIBLIOGRAFJA}

ACOSTA, D. A.; FACHJN T., A.; VILCHEZ R., 1.1992. Tortugas del género Podocnemis mantenidas en cautiverio en los alrededores de Iquitos, LoretoPerú. En: Boletín de Lima. 84:79-88.

1995. Alimentación de crías de taricaya Podocnemis unifilis (Chelonia:

Pelomedusidae) en cautiverio, Iquitos-Perú. En: Libro de Resúmenes del II Congreso Internacional sobre manejo de Fauna silvestre en la Amazonía. 712 mayo. Iquitos (Perú). pp. 7. 